\title{
OPTIMETA - Strengthening the Open Access publishing system through open citations and spatiotemporal metadata
}

\author{
Christian Hauschke ${ }^{\ddagger}$, Daniel Nüst§, Anette Cordts ${ }^{\ddagger}$, Svantje Lilienthall \\ ‡ TIB - Leibniz Information Centre for Science and Technology, Hannover, Germany \\ $\S$ Institute for Geoinformatics (ifgi), University of Münster, Münster, Germany \\ I TIB - Leibniz Information Centre for Science and Technology (formerly), Hannover, Germany
}

Corresponding author: Christian Hauschke (christian.hauschke@tib.eu)

Reviewable $\quad \vee 1$

Received: 22 Mar 2021 | Published: 14 Apr 2021

Citation: Hauschke C, Nüst D, Cordts A, Lilienthal S (2021) OPTIMETA - Strengthening the Open Access publishing system through open citations and spatiotemporal metadata. Research Ideas and Outcomes 7 : e66264. https://doi.org/10.3897/rio.7.e66264

\begin{abstract}
The BMBF project OPTIMETA aims to strengthen the Open Access publishing system by connecting open citations and spatiotemporal metadata from open access journals with openly accessible data sources. For this purpose, we will extend Open Journal Systems (OJS) to give it functionalities for collecting and distributing open data by developing two OJS plugins for capturing citation networks and articles' spatial and temporal properties as machine-readable and accessible metadata. We will ensure the target group-orientated design of the plugins by performing a comprehensive needs analysis for key stakeholders: the editors or operators of OA journals and the researchers, as authors and readers of articles. The developments will be designed and tested in cooperation with several independent journals and OA publishers. Overall, OPTIMETA supports the attraction of independent $O A$ journals as publication venues by substantially improving the discoverability and visibility of OA publications through enrichment and interlinking of article metadata.
\end{abstract}




\section{Keywords}

open access publishing, open research information, geospatial metadata, open citations

\section{Short description of the project}

The OPTIMETA project strengthens the Open Access (OA) ecosystem by capturing articles' citation information and spatiotemporal metadata and transferring these as open data from OA journals to openly accessible data sources. This work contributes to the metadata commons by adding crucial functionalities to Open Journal Systems (OJS), which is the most widely used free and open source software for publishing scientific journals. In this way, the aforementioned metadata can be collected, used by journals and shared with others to enable novel metascience studies and discovery of research artefacts. By combining both open source and open data, journals will be able to offer their respective communities innovative services for linking articles in a semantically meaningful way via geodata and to contribute to open citation graphs, such as $\mathrm{COCl}$ (OpenCitations Index of Crossref open DOI-to-DOI, Heibi et al. 2019a). Both geodata and citation data give publications better visibility and, thus, will increase findability, which in turn will increase the attraction of $O A$ journals as publication venues.

On the technical side, this project aims to develop two OJS plugins for spatiotemporal and citation metadata, respectively. With these plugins, authors and editors can create or extract the geographic and citation data during an article's publication process, validate it if necessary and transfer it to open, freely licensed data sources, such as Wikidata. Independent OA journals run by the scientific community, professional organisations or universities usually have few human and financial resources (Björk et al. 2016). Therefore, OPTIMETA will rely on automatic and semi-automatic processes for efficient and reliable gathering of metadata, suitable for even non-experts to use. In addition, we will focus on making the software user-friendly and sustainable, as well as adapting the application to the needs of the user groups (authors, editors, OJS hosters and OA publishers). This is ensured by a user-centred design and iterative, agile development processes in constant exchange with the future users of the project results and the OJS community and, in particular, with a group of partner journals.

\section{Idea and research questions}

An important part of a future OA ecosystem is a diverse publishing landscape with small and independent journals of high scientific quality. Transformating the landscape towards this vision will not only lead to a change in publishers' business and financing models, but also to stronger commitments by universities and scientists to help establish and operate independent journals and university publishers. Such a commitment is currently fuelled by a growing interest in supporting community-led OA journals (Prinčič and Belliard 2020) instead of commercial publishers, for example, who receive free labour from reviewers, yet it is also often constrained by limited human and financial resources. The editors of such 
OA journals seldom have the necessary funds for technical innovations and optimisations to improve the findability of the published works (Morrison 2016). To sustainably ensure their services, these independent journals must be able to offer their authors added value regarding both user-friendliness and scientific quality. They must also add value in terms of how their publications' metadata are collected, disseminated and even possibly reused.

On the basis of the Open Journal Systems (OJS, https://pkp.sfu.ca/ojs/) publication platform, which is used by more than 10,000 journals, OPTIMETA links OA articles with open data sources through systematic collection of metadata in the areas of spatiotemporal data and citation information. This approach promises to help scholars of all domains to more quickly discover both old and newly-published articles that are relevant for their areas and topics of interest, to work out relationships between research papers in metascience studies and to bring to light otherwise undiscoverable results. The project facilitates the representation of citation networks by including, where applicable, publications from OJS-based journals and research data referenced in the articles. In this way, the increased visibility of the published articles could eventually help bolster the reputations of the $\mathrm{OA}$ journals and their scientific authors and may spark new collaborations between otherwise disconnected researchers. OPTIMETA thus gives independent OA journals a technological edge over the commercially operated and mostly closed-off publication platforms.

The software that will be developed for this purpose primarily comprises two OJS plugins. First, the geoplugin records, displays and exposes the spatial extent and temporal scope of the underlying data or topics and issues in the articles. Second, the citation plugin extracts, checks and deposits citation information. Separating these two plugins by theme improves maintainability and reduces the entry hurdle for external developers to contribute to them, as well as for journals or publishers interested only in one of the two areas to install them.

The geoplugin supports authors by offering semi-automatic methods to capture an article's geographical and temporal metadata and to identify these metadata in a semantically unambiguous manner. By analysing and linking geodata, each article is assigned to one or more places or regions and one or more dates or time periods with which it has a direct or indirect relationship. This metadata is published in various machinereadable formats, for example, embedded in the article landing page and integrated into metadata exports. Map representations and visualisations of time intervals (Nüst et al. 2010) make spatiotemporal properties of individual articles quickly tangible and relate multiple articles to each other by putting them into context. These visualisations allow users to draw otherwise hidden connections and to make better individual decisions about interest and relevance. Overall, spatiotemporal metadata, as a specific kind of geodata, have integrative potential and are revolutionising a wide range of economic sectors (e.g. location-based services), but so far, they have been rarely used in scientific publications and in the OA ecosystem (Visser et al. 2020).

The citation plugin is dedicated to open citation information and thus specifically addresses the problem described by Heibi et al. 2019b, whereby the citations of smaller journals with limited technical and financial resources are missing from the Initiative for 
Open Citations (I4OC). This is problematic because the citation corpus on which scientometric indexes are based also determines the reputation of a journal. Particularly because highly cited journals are generally preferred as publication venues, the citation plugin can decisively contribute to improving the visibility of smaller OA journals.

In the course of developing and implementing the plugins, the FAIR principles (Findable, Accessible, Interoperable, Reusable; Wilkinson 2016) will be transferred to the enriched metadata of scientific articles and thus extended beyond their original scope of research data and their metadata. ${ }^{* 1}$

The OPTIMETA project bundles the considerations described in this work into three groups of research questions. The first group of questions focuses on the researchers, who play an essential role in the perceived quality of a journal with their decision to submit articles to the respective journal, contribute to the journal as reviewers or to cite work published in a journal. The two remaining questions address the journal side and take a look at scientific information systems from a higher-level perspective:

1. What criteria do researchers use to make a decision for or against a publication venue? Do "innovative metadata" influence this decision?

2. To what extent can the extraction of semantically meaningful metadata, especially citations and spatiotemporal metadata, be automated? Does a high level of automation or usability reduce editors' and publishers' efforts enough to raise their interest in using one or two of the plugins?

3. How can fully-fledged citation and geodata be integrated into scientific information systems? What are the limitations to and the potential benefits of better linking and discoverability of articles across individual platforms, for example, for innovative reuse?

\section{Project description}

\section{Objective of the project and addressing the purpose of the grant}

OPTIMETA extends the well-known publication platform for open access journals, OJS, with citation and spatiotemporal data for each article and thus improves the OA ecosystem on the metadata side. Two OJS plugins enable a large number of OJS-based OA journals to innovatively link publications via a significant improvement in metadata richness, diversity and quality. This strengthens the perceived quality of the publications, significantly increases their discoverability and thereby increases the attraction of small and independent journals. Overall, the project will enable numerous small and independent OA journals to contribute to establishing $\mathrm{OA}$ as the standard of scientific publishing, to improve the propagation of OA publications and to lead the way in innovative cooperations, based on free and open source software and open data. 


\section{Methodological approach to achieve the objective}

Various methods are combined to achieve the project's objectives. A mixed-methods approach will be used for the needs analysis. Expert interviews with editors and operators of small and independent journals, as well as researchers, will be used to identify key requirements for the design and features of OJS plugins. Later, a quantitative survey of these stakeholders will be conducted to determine their needs and priorities regarding the enrichment of article metadata with citation and geodata. These needs will then be formulated in user stories, on the basis of which the initial conception and the implementation of the two plugins will take place. In an iterative development process, a prototype will be made available to the community for testing and installation at selected cooperating partner journals ${ }^{*} 12$ will be actively supported. The feedback received during webinars and short surveys will flow back into the next iteration of conception and development. The development will take place in joint agile sprints of the partners. At the end of each sprint stands the release of a minimum viable product (MVP). The export of the citation and spatiotemporal metadata to the respective target systems will then be implemented in cooperation with the stakeholders. Dissemination and community outreach, for example, on the project website (https://projects.tib.eu/optimeta), project partner blogs and social media (https://twitter.com/optmta), will begin with the first contacts in the course of the needs analysis and will accompany the entire project. We will maximise probability of a broad uptake of the project results and the practice is maximised through the high quantity and quality of productive interactions with stakeholders (Wolf et al. 2013) and the publication of all developed software and documentation under open licences.

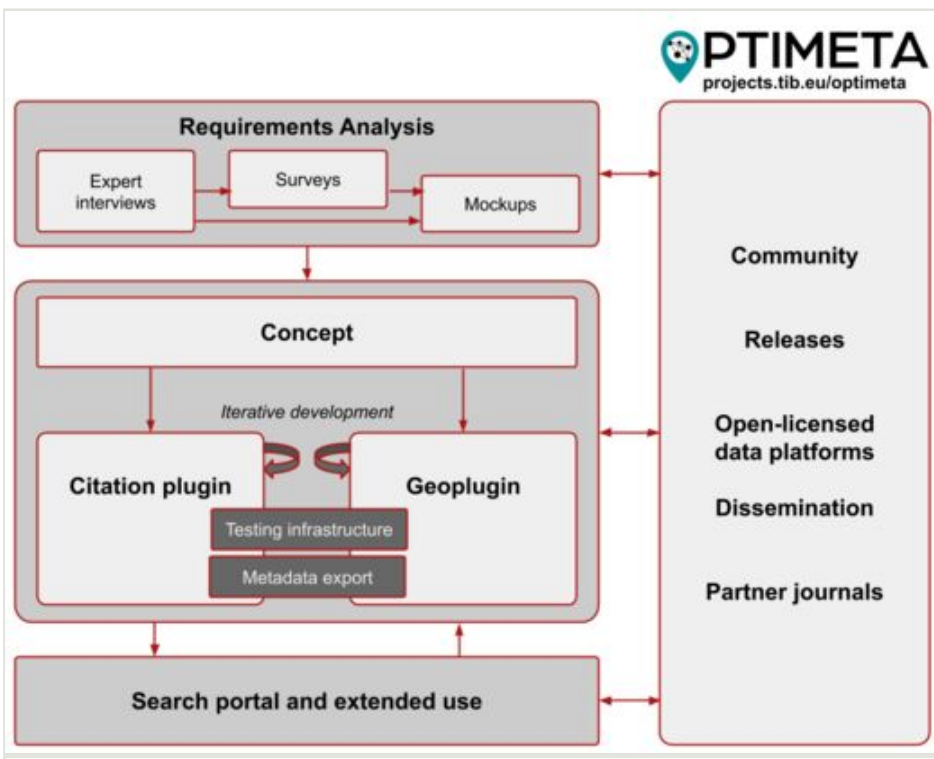

Figure 1. doi

Structure of the OPTIMETA project 


\section{State of research, technology and preliminary work}

This project can build on various initiatives, developments and preliminary work:

Open research metadata: The Initiative for Open Citations (I4OC) is an important initiative to promote structured and open scientific citation information. Other relevant initiatives include OpenCitations, DataCite Event Data, Crossref Event Data, WikiCite, the Crowdsour ced Open Citations Index ( $\mathrm{CROCl}$ ) and OpenAIRE. Citation metadata can be submitted via Cited-by (Crossref, DataCite). A general roadmap for "FAIR Research Information in Open Infrastructures" is currently being developed by the TIB and the State Scientific and Technical Library of Ukraine in the BMBF project FAIRIO.

Persistent identifiers: The PID graph (Fenner and Aryani 2019) developed in the EUfunded project FREYA is designed as a service within the framework of the PID infrastructure of the European Open Science Cloud (EOSC). With the PID graph, scientific output of any kind, for example, text publications, research data or software, can be identified by means of a Digital Object Identifier (DOI) and can link to other research output (DOI), researchers (ORCID), their funding (Grant ID, Brown 2019), their funders (Funder ID) and research organisations (ROR). It aims to improve the visibility, discoverability, transparency, reproducibility and quality control of research in line with the FAIR criteria. Persistent identifiers for academic events are currently being developed in the DFG project ConfIDent (TIB and RWTH Aachen University, Hagemann-Wilholt et al. 2020). In addition, the BMBF project Reference Implementation for Open Scientometric Indicators (ROSI, Hauschke et al. 2018) is based at TIB, in which open data sources for scientometric visualisations, based on PIDs, are being developed in OJS and VIVO (Conlon 2019). The Scholix initiative, called "A Framework for Scholarly Link eXchange", provides information on the links between scientific literature and research data, which are essentially exchanged between publishers and between repositories. Since Scholix enables interoperability between different PID metadata schemas, it can be used to trace data citations.

Data models for citation information: One of the data models for describing citation information is the Citation Typing Ontology (CiTO, Shotton 2010). Here, a citation can represent the linker that connects two publication entities, but the citation itself can also be its own entity. The Open Citation Identifier (OCl, Peroni and Shotton 2019) attempts to create a globally applicable and persistent identifier for identifying open citation information. InTRePID (In-Text Reference Pointer Identifier, Shotton 2020) offers another possibility for citation analysis. Lauscher et al. (2018) demonstrated how automatic reference extraction workflows can feed into an open citation database.

Geographic metadata for scientific papers: Linking publications, based on spatial and temporal contexts, helps to improve the availability of OA publications (Konkol and Kray 2018, Scheider et al. 2014, Katumba and Coetzee 2017, Degbelo et al. 2014) and helps to reduce biases in research coverage (Karl et al. 2013); but, unlike the documentation of data in curated repositories (Gil et al. 2016), linking aticles is not yet widespread. Previous approaches to derive geospatial metadata (georeferencing) from articles include text 
recognition (Kmoch et al. 2018) and pattern matching (Karl 2019). However, the majority of articles use only vague descriptions instead of coordinates, even when location is highly relevant for the work (Shapiro and Báldi 2012) and this was found to limit effective reuse of data and metadata, for example, in a synthesis study of case studies (Margulies et al. 2016). Multiple works have identified the need for standardisation of geospatial metadata (Kmoch et al. 2018), yet no common protocol exists to provide complete and well-defined geospatial metadata within scholarly publishing infrastructures. For example, the Dublin Core metadata standards have one loosely defined metadata element for geospatial and temporal information, namely the coverage element. In a broader effort, the Institute for Geoinformatics and the University and State Library of the WWU were early to promote the merging of scientific infrastructures and geographical information on the basis of Linked Open Data in the LODUM project and the experience gained from that project is being incorporated into OPTIMETA and expanded to include scientific publication systems. Finally, JournalMap (Karl et al. 2013) demonstrates the usefulness of geometric data in the area of ecology, but it is currently not a sustainable open infrastructure ${ }^{\star} 2$ and is also limited in functionality due to a simple single point geometry per article. A first prototype of the geoplugin that OPTIMETA will employ has been developed as part of a student thesis (Niers and Nüst 2020).

\section{Impact and scope of the results}

The OPTIMETA project's plan to extend the metadata commons will be implemented through the widely used, free software Open Journal Systems (OJS). Since the plugins will be developed bilingually in German and English and published in the OJS Plugin Gallery, all journals that use OJS will be able to benefit from them. In the German-speaking world alone, over 240 journals are based on OJS and, internationally, this number is more than 10,000 (OJS-de.net 2021). In addition, other open publication servers for scientific and scholarly work in OA could also profit from the project results: The overarching PKP ecosystem includes systems for preprints (Open Preprint Server, OPS) and monographs ( Open Manuscript Press, OMP), which rely on the plugin mechanisms, such that the plugins could potentially also be used in these platforms. In this way, a broad additional user group can be reached. The geoplugin is especially promising for OPS, in which spatiotemporal information could be used to better manage a continuous stream of new publications, whereas the citation plugin might be especially relevant for users of OMP. In these systems, the availability of the newly-developed functions would increase the reach of the project, thereby heightening the chances of long-term sustainable use of the plugins. In addition, with preprints and monographs, this project further supports important building blocks for establishing OA as a standard of scholarly publishing.

In principle, all scientific disciplines can benefit from the transmission of citation information and spatiotemporal metadata from OA journals to openly accessible data sources realised by OPTIMETA and, thus, also from the possibility of including citations in open citation graphs. In addition, almost all scientific work has a relation to space and time, whether related to locations of data collection or objects of study, such that the possibility of using 
geodata to identify articles and research would represent considerable added value for many research disciplines.

\section{Work planning}

This section contains a description of the project's work packages and division of labour. The work programme is divided into five work packages and extends over 24 months. In work package 1 (WP 1), we assess the needs of the stakeholder groups to define the framework conditions for conceiving and implementing the planned OJS plugins in the following work packages 2 to 4 . In work package 4, the developed OJS plugins are linked to external, open platforms and thus expanded in their application possibilities. Work package 5 focuses on public relations activities, as well as support for the partner journals.

\section{WP 1: Analysis of stakeholder needs}

\section{WP 1.1: Conduct a needs analysis via expert interviews and quantitative surveys}

Objectives: Survey of the requirements and expectations of the core target groups, namely (a) independent OA journals and university publishers (with a focus on Germanspeaking countries) and (b) academics from various research fields.

Tasks/approach: We will design, conduct and evaluate five to seven guided expert interviews with editors and operators of selected partner journals, as well as with researchers from different disciplines. This will allow us to collect qualitative information on the editors' requirements and needs regarding the extraction and transmission of citation and geodata. In addition to the already cooperating partner journals, other journals will be contacted and invited to participate. Based on the results of the expert interviews, we will design two quantitative online surveys for both core target groups, test them in a pre-test to be carried out at the TIB and then implement and evaluate them. The target number of participants in each case is 100 to 200 respondents.

\section{WP 1.2: Develop user stories and evaluate prototypes}

Objectives: Gather the requirements of the journals and researchers in the form of user stories and evaluate the first prototypical implementations of the plugins in terms of their functionalities and user experience (UX).

Tasks/approach: Based on the results of the expert interviews and the quantitative surveys, we will compile the requirements stated by the journals and researchers into user stories and collect and prioritise them in a public backlog. As soon as the first prototypical implementations of the plugins from WP 3 are available, they will be evaluated in an iterative procedure by conducting further expert interviews and at least one webinar and further requirements for the plugins will be determined. In addition to functionalities, the focus here will also be on UX: which validation steps are necessary before data is 
transferred to target systems? Which user group (authors, editors etc.) should perform them? Are the range of functions and the associated controls complete and easy to use? For more effective communication with stakeholders, a series of mock-ups are to be created. With the help of the mock-ups, users can better understand the functions and can simulate the work processes, so that any missing steps become visible and new aspects can be found. The mock-ups will be updated at least at the end of the needs analysis and assigned to the appropriate user stories. They will then serve as visual guidelines for the developers. The backlog will also be revised, if necessary, with updated user stories, based on the further interviews and the webinar.

\section{WP 2: Design and prepare for the technical implementation}

In work package 2, preparatory measures for the development of the two OJS plugins will be implemented. An overarching goal of the project is that the plugins will be stable and intuitive, as it is likely that users with a wide variety of backgrounds will need to install, administer and use the plugins and any faulty or complex installation processes may limit the distribution of the OJS extensions.

\section{WP 2.1: Design of the OJS plugins}

Objectives: Create a concrete conceptual design of the plugins.

Tasks/approach: On the basis of the survey in WP 1 and the project work planning, the technical and content-related foundations for the development of the plugins will be worked out in WP 2 and a technical implementation concept will be drawn up. This includes issues such as determining how exactly to integrate the plugins features into OJS, examining all hooks that are likely to be required and determining which extension options are available or missing in OJS. Furthermore, we will identify which functions require an extension of existing (default) plugins and contact will be made with the respective developers of plugins, for example, metadata export or core functions of OJS, for example, PKP staff.

\section{WP 2.2: Prepare for development}

Objectives: Prepare technical measures for plugin development.

Tasks/approach: In preparation for implementation, relevant OJS versions (determined via WP 1) will be installed on an internal test server at TIB. The test server will include the current development version and the latest release of OJS and will be organised in such a way that further versions, identified by the requirements analysis, can be added as needed. The use of containers ${ }^{*} 3$ is being investigated for the test server. For the two plugins, the software repositories on GitHub are prepared and populated with a rudimentary file structure for OJS plugins. These files include source code, documentation and test code so that new functions can be effectively developed from the beginning of the implementation phase. The developer documentation and test infrastructure reflect the technology used at OJS: Doxygen for documentation of the source code, as well as automated tests (continuous integration, $\mathrm{Cl}$ ) including "headless" browsers for user interface tests (UI tests) 
on Travis $\mathrm{Cl}$. The preparation of the plugin repositories will be completed by successfully running two exemplary unit tests each of the source code and a $\mathrm{UI}$ test on the $\mathrm{Cl}$ platform.

\section{WP 3: Implementation}

The implementation of the citation plugin and geoplugin will be done cooperatively by the partners. This large work package will be divided into manageable work stages using an agile, iterative development approach.

\section{WP 3.1: Citation plugin}

Objectives: Implement the citation plugin.

Tasks/approach: The OJS plugin for extracting, validating and submitting citation information to open citation indexes will be implemented in several steps. The first step deals with linking two documents with an assigned DOI. For this purpose, the DOI of the source document will be linked to further DOls located in the document and exported to Open Citations according to the $\mathrm{CROCl}$ procedure (Heibi et al. 2019b). In the next stage, transmission will be implemented according to the OpenCitations Ontology (OCO, Daquino and Peroni 2019). The necessary metadata will be obtained from OJS, mapped to OCO and validated. If validation by a user is successful, the metadata will be submitted to Open Citations. The third stage involves submitting the metadata to Wikicite, which is a community that maps information about article data in Wikidata. An automated process (a so-called bot) is being developed that will become active as soon as new citations are recorded. In addition to the developer-written automated tests, continuous verification will be performed by user testing, based on the results of the needs analysis.

\section{WP 3.2: Geoplugin}

Objectives: Implement the geoplugin.

Tasks/approach: First, we will implement the primary needs that can be realised by all supported OJS versions without installing additional software. These include the userdriven metadata collection of simple geometries on the basis of a web map 4 , the linking of geometries and place names 5 and the article- and journal-specific availability via embedding in $\mathrm{HTML}^{*} 6$, via the OJS API*7, via integration in metadata exports*8 and via the cartographic or graphical representation of the spatial and temporal extent of individual articles and all articles of a journal and in a timeline. In a further step, we will implement a geodata extraction and extension of the search functions, which will require installing additional software or connections to specific services to leverage geospatial database indexing. The automatic extraction supports uploaded geodata* 9 , links to selected services ${ }^{*} 10$ and derives geodata via the title and abstract of the article with a gazetteer. The advanced search functions will use spatial indices*11 for spatial and temporal restriction of results in article searches. The functions of both steps will be realised in the same plugin and coordinates will be stored and published exclusively in the WGS84 coordinate system, which provides sufficient accuracy for finding articles. Just as with the 
citation plugin, the automated tests, written by developers, will be complemented by continuous user testing, based on the results of the needs analysis.

\section{WP 3.3: Finalisation, rollout and release of plugins}

Objectives: Fixing bugs and releasing versions of single or both plugins as required.

Tasks/approach: Self-discovered or user-identified bugs will be fixed and other user feedback will be incorporated into the plugins as needed. Usability and user experience will be optimised on the basis of ongoing use by the partner magazines. Releases of the plugins will be published as required and, eventually, a final release will be prepared, extensively tested and published in a suitable repository that offers the publication to be citable and available over the long-term, for example, Zenodo. The releases in the OJS Plugin Gallery will be accompanied by in-depth testing by the PKP developers, which will be organised and accompanied in this WP.

\section{WP 4: Export, search portal and extended use}

\section{WP 4.1: Metadata export to openly-licensed data platforms}

Objectives: Publish the collected metadata in openly-licensed data platforms.

Tasks/approach: In this WP, we will test both of the plugins' connection to different open data platforms with overlapping functionality and implement them in a common code base (M 4.1). For Wikidata, for example, we will need to store geodata as point data (centroid of more complex geometries). Other target systems are Crossref, DataCite, the PID graph and the Open Research Knowledge Graph (ORKG).

\section{WP 4.2: Search portal}

Objectives: Design and implement a search portal for OA articles, based on spatiotemporal metadata.

Tasks/approach: In this WP, as a proof of concept, we will conceptualise and implement a prototype of a search portal that aggregates and makes accessible metadata from different OJS-based journals, based on the plugins we created. The search portal provides (a) an intuitive map display of articles from journals across many OJS servers and (b) the possibility for users to subscribe to new publication alerts for specific areas. The portal demonstrates the advantages and opportunities that geospatial data can provide for the discoverability of OA articles. Possible architectures under consideration are a document database that uses a spatial search (e.g. Elasticsearch) and that operates based solely on the open data and its APIs. For the regular collection of data (so-called harvesting) for the document database, both the public target systems (see WP 4.1) and the variants of metadata publication implemented in WP 2 (API, embedded metadata in HTML etc.) will be tested and thus validated. Subscriptions will be implemented in a minimalist way using emails about global time intervals, for example, all articles from the last month at the end of 
the month, for spatial and temporal sections defined by the user. The user accounts will be managed with passwordless authentication via email. An established standard software for an HTML5-based user interface and a server component will be used (e.g. Leaflet.js, Django), which communicate via an internal RESTful API. The search portal will be installed in the WWU cloud and operated in a publicly accessible manner at least until the end of the project. In addition, we will explore the technical and organisational sustainability of the implementation, for example, by incorporating the functionalities of the search portal into library catalogue or discovery systems.

\section{WP 4.3: Extended use}

Objectives: Implement the plugins in other systems.

Tasks/approach: The plugins will be ported and tested in systems that share a code base with OJS: Open Preprint Server (OPS) and Open Manuscript Press (OMP). For this purpose, test datasets with at least 10 articles or manuscripts will be created and entered into local developer installations. Errors or missing functions or workflows that are not covered by the plugins will be corrected within the available time budget or documented in the form of issues with a common tag ("OMP" or "OPS") in the repositories of the plugins if they exceed the time budget. If the plugins are successfully ported, operators of OMP or OPS servers will be contacted and supported in an evaluation of the plugins.

\section{WP 5: Dissemination and support}

In WP 5, all work results will be documented for easy reuse and the resulting source code and anonymised raw data of the standardised surveys will be published. In addition, close cooperation will be maintained with a number of partner journals which, at this time, will operate the plugins in full functionality.

\section{WP 5.1: Publications and public relations}

Objectives: Engage in target group-orientated public relations, taking into account all stakeholder groups and publish scientific and practice-orientated articles.

Tasks/approach: The project and its current status will be documented on a project website. The developed plugins will be presented at community events (Open-Access-Tag e, German-language OJS Developer Workshop, PKP Conference, Bibliothekartag, OASPA Conference) and in practice-orientated online publications. To maintain communication with stakeholders and for further dissemination, webinars will be organised for OJS operators and users, including OJS journals that are not partner journals and especially other journals related to geospatial data, as they are potential users of the geoplugin. The Wikicite and Wikidata communities will be kept informed about the planning and development in order to create awareness around the citation plugin. The results of the project will be documented for future use, which will include presenting the plugins and survey results in typical community communication tools, such as blogs or the PKP forum. In addition, scientific contributions in Open Access publication formats will be prepared and 
submitted on the basis of the project results. Furthermore, a final workshop with OJS users and operators, as well as members of other stakeholder groups, will be organised at TIB in order to improve the follow-up use of the project results. Finally, a summary report will be prepared.

\section{WP 5.2: Support partner journals and documentation}

Objectives: Smoothly install and apply the plugins at the partner journals.

Tasks/approach: The partner journals will be supported by the project staff with installing a pre-release version (at least one partner journal) and the releases of the OJS plugins from the OJS Plugin Gallery (eventually all partner journals). For the first operators, this support will be provided on site and will ideally be combined with a meeting with users, for example, editors and journal managers, to improve the documentation and collect feedback. In addition, manuals on how to handle geodata and citation information will be prepared and given to the journal operators. The user documentation for end users (authors, editors) will also be revised in consultation with selected stakeholders. Furthermore, at the end of the second development phase, all stakeholders of the partner journals, i.e. authors, reviewers, editors and managers, will be invited to participate in an online survey to gather broad feedback on the use of the plugins.

\section{Tabular overview of the scope of work and schedule}

Table 1.

Overview of workloads

\begin{tabular}{|c|c|c|}
\hline $\begin{array}{l}\text { WP } \\
\text { Nr. }\end{array}$ & Task sharing & $\begin{array}{l}\text { workload } \\
\text { (PM) }\end{array}$ \\
\hline 1 & $\begin{array}{l}\text { The TIB carries out the requirements analysis using different methods and involving the various } \\
\text { external partners and experts. }\end{array}$ & TIB: 9 \\
\hline 2 & $\begin{array}{l}\text { The technical conception of the plugins and transfer of the user stories into implementation tasks } \\
\text { is carried out jointly by TIB and WWU. The TIB prepares the basic development and test } \\
\text { infrastructure and begins as early as possible with the preparatory testing of the basic software } \\
\text { as well as related projects and external communication. }\end{array}$ & $\begin{array}{l}\text { TIB: } 3 \\
\text { WWU: } 2\end{array}$ \\
\hline 3 & $\begin{array}{l}\text { The WWU is in charge of developing the geoplugin, the TIB the citation plugin. Both institutions } \\
\text { are involved in the development of the other plugin to a certain degree in order to ensure a } \\
\text { sustainable and effective development and to use synergy effects. The TIB supports the testing } \\
\text { of releases by another project staff member and is also responsible for troubleshooting and, if } \\
\text { necessary, further development of the plugins during testing after the end of the WWU sub- } \\
\text { project, as well as for the releases. }\end{array}$ & $\begin{array}{l}\text { TIB: } 7 \\
\text { WWU: } 6\end{array}$ \\
\hline 4 & $\begin{array}{l}\text { The TIB and the WWU jointly realise the shared code base for overlapping export functions of } \\
\text { the plugins and the porting of the plugins to other potentially wide-reaching publication platforms. } \\
\text { The WWU develops an implementation concept and a prototype for a cross-journal geodata- } \\
\text { based search portal with simultaneous testing of harvesting variants. }\end{array}$ & $\begin{array}{l}\text { TIB: } 1 \\
\text { WWU: } 3\end{array}$ \\
\hline
\end{tabular}




\begin{tabular}{|l|l|l|}
\hline $\begin{array}{l}\text { WP } \\
\text { Nr. }\end{array}$ & Task sharing & $\begin{array}{l}\text { workload } \\
\text { (PM) }\end{array}$ \\
\hline 5 & $\begin{array}{l}\text { The TIB is responsible for the sustainable dissemination and communication of the project } \\
\text { results in various forms of publication, especially in documentation, scientific publications and } \\
\text { project reports, as well as in events, especially webinars and the final workshop. The WWU } \\
\text { supports the writing of publications and participates in events. WWU and TIB jointly support the } \\
\text { partner journals including on-site meetings. }\end{array}$ & $\begin{array}{l}\text { TIB: } 10 \\
\text { WWU: } 1\end{array}$ \\
\hline
\end{tabular}

\section{Exploitation and reuse of the results}

The results of this project open up a variety of possibilities for further use (see also WP 5.1). In addition to the publication of the project results in scientific OA journals, other online publications are planned that should help to make the tools developed in the project known to the professional public. A dedicated website, https://projects.tib.eu/optimeta, will be set up to serve different target groups and will offer examples on how to contribute to the metadata commons and thus to the promotion of OA.

The results will continue to be used by peer-reviewed OA journals or journal platforms as external partners of OPTIMETA*12. These partner journals will install the current OJS plugins in at least two points in time, test the functionality with their various user groups, support the requirements analysis and report errors during operation. One focal point in the place of publication of the partner journals is Germany and other journals have already signalled their interest in cooperation. At the TIB, the developed OJS plugins will be recommended for use by the editors of journals hosted there and will be installed free of charge. In addition, the transmission of geodata to the ORKG is planned. Furthermore, the metadata enrichments that OPTIMETA will offer will be incorporated into the PID graph by means of DOI-to-DOI linking.

The citation information and geodata obtained during the project will be collected and published under a free licence with the granting of the broadest possible rights of use, Crea tive Commons Zero (CCO 1.0). This enables (a) the professional public to use the data developed in this project as a basis for further research and (b) publishers to include it in their own services. Through detailed documentation and software testing, individual journals or publishers can further develop the OJS plugin. In line with practice in the OJS community, the GNU GPL version 3 will be used for software. Texts and graphics will be published under a Creative Commons Attribution 4.0 International (CC BY 4.0) licence and research data will be dedicated to the public domain under CCO 1.0 where possible.

\section{Budget plan}

A total of $€ 296 k$ has been requested (TIB: $€ 197 k$, WWU: $€ 99 k$ ). The TIB will provide the project management and the advisory support in the course of the development and implementation of the OJS plugins. Furthermore, the TIB will carry out the installation of 
the plugins to the journals hosted by the TIB, the hosting of the project website and the provision of facilities for a final workshop.

\section{Acknowledgements}

We would like to thank Tom Niers (WWU), Franziska Altemeier, Dulip Withanage and Simone Cartellieri (TIB) for their helpful feedback for this application and Celeste R. Brennecka from the Scientific Editing Service of the University of Münster for her editorial support. This project would not be possible without the support of our partners: Journal of Spatial Information Science; Kommunikations- Informations-, Medienzentrum (KIM), Universität Konstanz; WWU E-Journals, Universitäts- und Landesbibliothek Münster; ejournals Julius Kühn-Institut; Public Knowledge Project; Archäologischer Anzeiger (AA), Deutsches Archäologisches Institut (DAI); heiJOURNALS and Universitätsbibliothek Heidelberg/Universität Heidelberg.

\section{Funding program}

OPTIMETA is funded by the German Federal Ministry of Education and Research (BMBF) within the guidelines for funding projects to accelerate the transformation to Open Access (Förderung von Projekten zur Beschleunigung der Transformation zu Open Access, Bundesministerium für Bildung und Forschung 2020).

OPTIMETA is funded by the German Federal Ministry of Education and Research (BMBF) within the guidelines for funding projects to accelerate the transformation to Open Access (Förderung von Projekten zur Beschleunigung der Transformation zu Open Access).

\section{Conflicts of interest}

The authors declare that there are no conflicts of interest.

\section{References}

- Björk BC, Shen C, Laakso M (2016) A longitudinal study of independent scholarpublished open access journals. PeerJ 4 https://doi.org/10.7717/peerj.1990

- Brown J (2019) Crossref grant IDs: a global, open database of funding information and identifiers. Autumn 2019 euroCRIS Strategic Membership Meeting. Strategic Membership Meeting 2019 - Autumn, Münster, Nov 18-20, 2019. euroCRIS, 33 pp. URL: http://hdl.handle.net/11366/1249

- Bundesministerium für Bildung und Forschung (2020) Richtlinie zur Förderung von Projektenzur Beschleunigung der Transformation zu Open Access. Bundesanzeiger, Bundesministerium der Justiz und für Verbraucherschutz. URL: https://www.bildungforschung.digital/files/BAnz\%20AT\%2017.06.2020\%20B3-1.pdf 
- Conlon M, et al. (2019) VIVO: a system for research discovery. The Journal of Open Source Software 4 (39). https://doi.org/10.21105/joss.01182

- Daquino M, Peroni S (2019) OCO, the OpenCitations Ontology. https://opencitations.github.io/ontology/current/ontology.html.

Accessed on: 2021-2-08.

- Degbelo A, Kuhn W, Przibytzin H, Scheider S (2014) Content and context descriptionHow linked spatio-temporal data enables novel information services for libraries. gis.Science 4: 138-149. URL: https://www.semanticscholar.org/paper/Content-andcontext-description-How-linked-data-for-Degbelo-Kuhn/ b31adbb47d54bee3c020c3b374ca14e69d1186aa

- Fenner M, Aryani A (2019) Introducing the PID Graph. (Version 1.0). https://doi.org/ 10.5438/JWVF-8A66

- Gil Y, David CH, Demir I, et al. (2016) Toward the Geoscience Paper of the Future: Best practices for documenting and sharing research from data to software to provenance. Earth and Space Science 3 (1): 388-415. https://doi.org/10.1002/2015EA000136

- Hagemann-Wilholt S, Plank M, Hauschke C (2020) ConfIDent - An Open Platform for FAIR Conference Metadata. In: Farace D, Frantzen J (Eds) Open Science Encompasses New Forms of Grey Literature. Twenty-First International Conference on Grey Literature, Hannover, 22/23.10.2019. TextRelease, Amsterdam, 5 pp. https://doi.org/10.15488/9424

- Hauschke C, Cartellieri S, Heller L (2018) Reference implementation for open scientometric indicators (ROSI). Research Ideas and Outcomes 4 https://doi.org/ 10.3897/rio.4.e31656

- Heibi I, Peroni S, Shotton D (2019a) Software review: COCl, the OpenCitations Index of Crossref open DOI-to-DOI citations. Scientometrics 121 (2). https://doi.org/10.1007/ s11192-019-03217-6

- Heibi I, Peroni S, Shotton D (2019b) Crowdsourcing open citations with CROCI. An analysis of the current status of open citations, and a proposal. URL: https://arxiv.org/ abs/1902.02534

- Karl J (2019) Mining location information from life- and earth-sciences studies to facilitate knowledge discovery. Journal of Librarianship and Information Science 51 (4): 1007-1021. https://doi.org/10.1177/0961000618759413

- $\quad$ Karl JW, Herrick JE, Unnasch RS, Gillan JK, Ellis EC, Lutters WG, Martin LJ (2013) Discovering Ecologically Relevant Knowledge from Published Studies through Geosemantic Searching. BioScience 63 (8). https://doi.org/10.1525/bio.2013.63.8.10

- Katumba S, Coetzee S (2017) Employing Search Engine Optimization (SEO) Techniques for Improving the Discovery of Geospatial Resources on the Web. ISPRS International Journal of Geo-Information 6 (9): 284. https://doi.org/10.3390/ijgi6090284

- Kmoch A, Uuemaa E, Klug H, Cameron S (2018) Enhancing Location-Related Hydrogeological Knowledge. ISPRS Int. J. Geo-Inf 7 (4). https://doi.org/10.3390/ ijgi7040132

- Konkol M, Kray C (2018) In-depth examination of spatiotemporal figures in open reproducible research. Cartography and Geographic Information Science 46 (9): 412-427. https://doi.org/10.1080/15230406.2018.1512421

- Lauscher A, Eckert K, Galke L, Scherp A, Rizvi STR, Ahmed S, Dengel A, Zumstein P, Klein A (2018) Linked Open Citation Database. Proceedings of the 18th ACM/IEEE on Joint Conference on Digital Libraries https://doi.org/10.1145/3197026.3197050 
- Margulies J, Magliocca N, Schmill M, Ellis E (2016) Ambiguous Geographies: Connecting Case Study Knowledge with Global Change Science. Annals of the American Association of Geographers 106 (3): 572-596. https://doi.org/ 10.1080/24694452.2016.1142857

- Morrison H (2016) Small scholar-led scholarly journals: Can they survive and thrive in an open access future? Learned Publishing 29 (2). https://doi.org/10.1002/leap.1015

- Niers T, Nüst D (2020) Geospatial Metadata for Discovery in Scholarly Publishing. The 15th Munin Conference on Scholarly Publishing 2020. 15 th Munin Conference on Scholarly Publishing 2020, November 17-19, 2020. Septentrio Conference Series https://doi.org/10.7557/5.5590

- $\quad$ Nüst D, Bache F, Bröring A, Sasch C, Jirka S (2010) Visualizing the Availability of Temporally Structured Sensor Data. In: Painho M, Santos MY, Pundt H (Eds) Short Paper Proceedings of the 13th AGILE International Conference on GIScience 2010. 13th AGILE International Conference on GIScience 2010, Guimarães, Portugal. https://doi.org/10.31223/osf.io/jq5df

- $\quad$ OJS-de.net (2021) Über OJS. https://ojs-de.net/ueber-ojs. Accessed on: 2021-2-05.

- Peroni S, Shotton D (2019) Open Citation Identifier: Definition. Figshare. https://doi.org/ 10.6084/m9.figshare.7127816

- $\quad$ Prinčič A, Belliard F (2020) Towards a community-driven, open access university publisher. Research Information URL: https://www.researchinformation.info/analysisopinion/towards-community-driven-open-access-university-publisher

- $\quad$ Scheider S, Degbelo A, Kuhn W, Przibytzin H (2014) Content and context description How linked spatio-temporal data enables novel information services for libraries. gis.Science 4: 138-149.

- $\quad$ Shapiro JT, Báldi A (2012) Lost locations and the (ir)repeatability of ecological studies. Frontiers in Ecology and the Environment 10 (5): 235-236. https://doi.org/ 10.1890/12.WB. 015

- $\quad$ Shotton D (2010) CiTO, the Citation Typing Ontology. Journal of Biomedical Semantics 1 (Suppl 1). https://doi.org/10.1186/2041-1480-1-S1-S6

- $\quad$ Shotton D (2020) In-Text Reference Pointer Identifiers - InTRePIDs. PIDapalooza URL: https://pidapalooza20.sched.com/event/XIAA

- $\quad$ Visser M, van Eck NJ, Waltman L (2020) Large-scale comparison of bibliographic data sources: Scopus, Web of Science, Dimensions, Crossref, and Microsoft Academic. URL: https://arxiv.org/abs/2005.10732

- Wilkinson M, et al. (2016) The FAIR Guiding Principles for scientific data management and stewardship. Scientific Data 3 https://doi.org/10.1038/sdata.2016.18

- Wolf B, Lindenthal T, Szerencsits M, Holbrook JB, Heß J (2013) Evaluating Research beyond Scientific Impact. How to Include Criteria for Productive Interactions and Impact on Practice and Society. GAIA - Ecological Perspectives for Science and Society 22 (2): 104-114. https://doi.org/10.14512/gaia.22.2.9 


\section{Endnotes}

*1 This takes up the idea of the BMBF-funded project FAIRIO currently running at TIB, which applies the FAIR principles to research information: German National Library of Science and Technology (n.d.): FAIRIO. FAIR Research Information in Open Infrastructures. https://projects.tib.eu/fairio

*2 Even if an API exists and there are cooperations with publishers, the JournalMap is to be seen as a "data sink" and not an open infrastructure (download of data "coming soon", as well as a "non commercial" data licence) and, therefore, not a target system for this application.

*3 Based on the OJS Docker project (beta development status): https://github.com/pkp/ docker-ojs/

*4 Points, lines, polygons; acquisition based on Leaflet: https://leafletjs.com/

*5 Proposing (standardised) geometries and names via gazetteers (e.g. GeoNames, https://www.geonames.org/), databases with administrative boundaries (e.g. GADM, https://gadm.org/) and standards data (e.g. Gemeinsame Normdatei - GND, https://www.dnb.de/DE/Professionell/Standardisierung/GND/gnd node.html).

*6 E.g. via microformats (http://microformats.org/wiki/h-geo) and elements of Dublin Core (Dublin Core 'Coverage' is already rudimentarily implemented as a simple text field in the Dublin Core Meta Plugin, but the use of 'Spatial Coverage' and 'Temporal Coverage', possibly even DCLite4G (https://wiki.osgeo.org/wiki/DCLite4G) should be checked here) and Schema.org (https://schema.org/GeoShape).

*7 Cf. Public Knowledge Project (2020a): REST API Usage Guide.

https://docs.pkp.sfu.ca/dev/api/; Encoding e.g. as GeoJSON or Linked Open Data in Turtle (cf. Wikipedia (2020): Turtle (Syntax). In: Wikipedia, The Free Encyclopedia. https://de.wikipedia.org/wiki/Turtle (Syntax)).

*8 E.g. OAI-PMH (via Dublin Core) and native XML.

*9 The Python library geoextent (https://o2r.info/geoextent/, developed at WWU) can derive geographic and temporal extents from typical geodata formats (e.g, GeoJSON, NetCDF, Shapefile)

*10 Services considered are data repositories, for example, PANGEA

(https://www.pangaea.de/) or GFZ Data Services (https://dataservices.gfzpotsdam.de/), catalogues, for example, CKAN (https://ckan.org/portfolio/geospatial/) or OGC CSW (https://de.wikipedia.org/wiki/Web Catalogue Service) and spatial data servers, for example, OGC W\{C,F\}S (https://de.wikipedia.org/wiki/Web Feature Service, https://de.wikipedia.org/wiki/Web_Coverage Service).

*11 PostGIS (https://postgis.net/) for PostgreSQL, spatial data types in MySQL (https://dev.mysql.com/doc/refman/5.7/en/spatial-types.html) and Spatial Search for the OJS Lucene Plugin (https://lucene.apache.org/solr/guide/6 6/spatial-search.html; https://github.com/ojsde/lucene) will be evaluated.

*12 See full list of partner journals and platforms at https://projects.tib.eu/optimeta/en/. 